\begin{tabular}{|c|l|}
\hline Title & Formation mechanism of super water-repellent fractal surfaces of al kylketene dimer \\
\hline Author(s) & Fang, Wenjun; May ama, Hiroyuki; Tsujii, Kaoru \\
\hline Citation & $\begin{array}{l}\text { Colloids and Surfaces A : Physicochemical and Engineering A spects, 316(1-3), 258 265 } \\
\text { https://doi.org/10.1016/.colsurfa.2007.09.010 }\end{array}$ \\
\hline Issue Date & 2008-03-05 \\
\hline Doc URL & http://hdl.handle.net/2115/32660 \\
\hline Type & article (author version) \\
\hline File Information & tsuji.pdf \\
\hline
\end{tabular}

Instructions for use 


\title{
Formation mechanism of super water-repellent fractal surfaces of
}

\section{alkylketene dimer}

Wenjun Fang, ${ }^{\dagger a}$ Hiroyuki Mayama ${ }^{a}$ and Kaoru Tsujii* ${ }^{a, b}$

${ }^{a}$ Nanotechnology Research Center, Research Institute for Electronic Sciences, Hokkaido

University, N21, W10 Kita-ku, Sapporo 001-0021, Japan.

${ }^{\mathrm{b}}$ CREST, JST, Tokyo, Japan.

Corresponding author. Tel.: +81 11706 9456; fax: +81 11706 9357. E-mail address:

tsujik@es.hokudai.ac.jp (K. Tsujii)

$\dagger$ Present address: Department of Chemistry, Zhejiang University, Hangzhou 310027, China

\begin{abstract}
Alkylketene dimer (AKD), a kind of wax, has been known to form fractal surfaces spontaneously, and to show super water-repellent property. In order to understand further the super water-repellency of the AKD surfaces and to elucidate the mechanism of spontaneous formation of the fractal structures on the wax surfaces, a pure and a mixed AKD samples were employed to make the surfaces by the melt-solidification method. Time-dependent contact angles of water droplets on the AKD surfaces cured at several temperatures were systematically measured. It was found that spontaneous formation of
\end{abstract}


super water-repellent surfaces was thermally induced quite effectively. For example, it took about 6 days for the pure AKD surface to show the super water-repellency at $40{ }^{\circ} \mathrm{C}$ but did just 1 hour at $50{ }^{\circ} \mathrm{C}$. The fractal dimension of the mixed AKD surface with super water-repellency was calculated to be 2.26 from the SEM images by the box-counting method which should be reasonably compared with 2.29 of pure AKD surface. The mechanism for the spontaneous formation of fractal structures was discussed from the results of the time-dependent contact angles, differential scanning calorimetric (DSC) curves and X-ray diffractometric (XRD) patterns. It has been made clear that the fractal AKD surface and its super water-repellency result from the phase transformation from the metastable state to the stable one of the AKD crystals.

Key words: Super water-repellency; Fractal surface structures; Alkylketene dimer; phase transition 


\section{Introduction}

Super water-repellent or superhydrophobic surfaces having water contact angles larger than $150^{\circ}$ have attracted wide interestes because of the importance in chemical and biological researches and the potential applications in industry. ${ }^{1-10}$ In order to fabricate such surfaces, different materials and many methods have been introduced mainly by taking two factors, the surface energy and the surface roughness, into account. In the former trial, fluorinated compounds with low surface energy were usually used, while several nano- or micro-textured or fractal structures on the surfaces were formed in the later case. Various approaches to prepare super water-repellent surfaces have been reported including melt-solidification of a wax, ${ }^{11-14}$ silica and oxidized alumina films through the sol-gel method, ${ }^{15-17}$ plasma-based etching or deposition, ${ }^{18,19}$ gel-like roughened polypropylene, ${ }^{20}$ anodically oxidized aluminum, ${ }^{21,22}$ etched copper and electrodeposited copper, ${ }^{23}$ electrochemical polymerization, ${ }^{24}$ layer-by-layer assembly together with electrodeposition, ${ }^{25}$ solution-immersion, ${ }^{26}$ polymer/nanoparticle composite films, ${ }^{27,28}$ films of aligned carbon nanotubes, polymer nanofibers and inorganic nanorods. ${ }^{29-36}$ Effects of geometric length scales, surface roughness and surface energy on the wettability of super water-repellent surfaces were extensively discussed from physical and chemical points of view. ${ }^{37-45}$

It has been well known that fractal structures can effectively magnify the real surface area compared with the projected one, and then enhance the wettability of a solid surface. ${ }^{11-13,21,22,46}$ Alkylketene dimer (AKD), a kind of wax, was found to form fractal 
structures spontaneously with a dimension of 2.29 and to give super water-repellent surfaces with the water contact angle as large as $174^{\circ} .{ }^{11,12}$ Super water- and oil-repellent surfaces ${ }^{21,22}$ with fractal dimension of 2.19 and fractal super water-repellent poly(alkylpyrrole) film ${ }^{24}$ with a dimension of 2.23 were also successfully made. Because of the fractal structures and super water-repellent properties, the AKD surfaces have attracted much attention., ${ }^{1,11,12,14}$ Cell cultures on fractal super water-repellent AKD surfaces were recently introduced. ${ }^{47}$ The effects of some surfactants on the wettability of super water-repellent AKD surfaces were also investigated. ${ }^{14}$ The studies on the structure, property and application of the AKD surfaces provide much insights to explain theoretically the origins of super water-repellecy of the leaves of plants or insects in nature. ${ }^{47}$ Although the fractal structures and the super water-repellency of the AKD surfaces have been well-known and some biological or bionic applications were explored, the mechanism for spontaneous formation of the fractal structures on the AKD surfaces is not yet made clear.

In our recent work, ${ }^{13}$ the spontaneous formation of fractal structures on the wax surfaces of triglycerides, trimyristin, tripalmitin and tristearin, and their super water-repellent property have been studied. The spontaneous formation of the fractal structures on the triglyceride surfaces has been found to be originated from the phase transition from the metastable $\alpha$-form to the stable $\beta$-form crystal. As a continuation of our systematic studies on the AKD surfaces, the present work deals with the mechanism of spontaneous formation of super water-repellent AKD surfaces with fractal structures. If the mechanisms for the 
sponteneous formation of fractal surfaces of these two kinds of wax (AKD and triglyceride) are the same, we may be able to obtain a general guiding principle to make the fractal structure which is a powerful tool to develop some functional materials. ${ }^{48}$

\section{Experimental Section}

\subsection{Materials}

The pure alkylketene dimer (AKD), Fig.1, was purchased from Dojindo Laboratories, Japan and used without further purification. They synthesized the AKD sample especially for us according to our recipe. ${ }^{12}$ A typical procedure was as follows. $n$-octadecanoic acid chloride of $60.6 \mathrm{~g}$ dissolved in $100 \mathrm{ml}$ toluene was added dropwise to a solution of triethylamine of $22.3 \mathrm{~g}$ in $300 \mathrm{ml}$ toluene at $323 \mathrm{~K}$ with stirring in a glass flask. After the reaction finished, the by-product of triethylamine hydrochloride was filtered off at $323 \mathrm{~K}$. Crude AKD sample was obtained with weak brown color by evaporating the solvent in vacuum. The crude AKD was purified with a silica-gel (C200;WAKO) column using the chloroform/n-hexane mixtures having the gradients of $0 / 100$ to $50 / 1$ as the elution solvent. Pure AKD (m.p. $=66-67{ }^{\circ} \mathrm{C}$ ) was obtained as the early fractions of the elute. The final sample of the AKD was checked to be more than $98 \%$ pure by gas chromatography. Main impurity still remained was the dialkylketone. The mixed AKD wax was kindly presented by Arakawa Chemical Industries, Ltd., Japan. It was synthesized from a mixture of 
$n$-hexadecanoic $(30-40 \mathrm{~mol} \%)$ and $n$-octadecanonic acid $(70-60$ mol \%) chlorides utilizing essentially the same procedures as those mentioned above. The mixed AKD sample was purified by recrystallization twice from $n$-hexane. The melting point of the mixed AKD was around $45{ }^{\circ} \mathrm{C}$ depending upon the mixing ratio of octadecanoic and hexadecanoic acid chloride.

\subsection{Preparation of sample surfaces}

A small amount of the pure or the recrystallized mixed AKD was put on a polystyrene plate $(40 \mathrm{~mm} \times 40 \mathrm{~mm} \times 1 \mathrm{~mm})$ and heated slightly above its melting point $\left(c a .66{ }^{\circ} \mathrm{C}\right.$ for the pure AKD or $53{ }^{\circ} \mathrm{C}$ for the mixed one) on an electric hot plate. Solidification was then carried out from the melt by moving the sample quickly onto a cold $\left(c a .0{ }^{\circ} \mathrm{C}\right)$ plate to obtain the fresh solid AKD surface. The thickness of the AKD surface was $200 \sim 300 \mu \mathrm{m}$. After the initial contact angle for water was measured, the sample was transferred to an incubator (FF-30N, Tokyo Glass Machinery Co. Ltd.) for heat-treatment for a given time at a specified temperature.

\subsection{Analytical measurements}

Time-dependent contact angles on the AKD surfaces were measured with an optical contact angle meter (DropMaster 300, Kyowa Interface Science Co. Ltd.) at room temperature. Two surface samples were prepared for each measurement. Water droplets of 3 
$\mu \mathrm{L}$ were placed on ten different positions of each surface and the contact angles were measured. The averaged value was adopted as the reported contact angle. The ultrapure water with the resistivity above $18.0 \times 10^{6} \Omega \cdot \mathrm{cm}$ at $25{ }^{\circ} \mathrm{C}$ produced by the Milli-Q system was used.

The AKD surfaces and their cross sections were observed by using a field-emission scanning electron microscope (FE-SEM, S-5200, Hitachi). Prior to the SEM observations, the AKD film was set onto an aluminum sample stage and coated with a thin layer of sputtered alloy of gold and palladium by using the ion sputter (E-1030, Hitachi). The SEM images at several magnifications of the cross sectional view of the super water-repellent AKD surface were employed to evaluate the fractal dimension.

Phase transformations in the AKD samples were detected by differential scanning calorimetry (DSC) and X-ray diffractometry (XRD). The DSC measurements were performed on a Thermo Plus 2 differential scanning calorimeter (DSC-8230, Rigaku). The sample size was $3 \sim 4 \mathrm{mg}$. It was sealed in an aluminum pan and a similar empty pan was used as a reference. Each sample was scanned for two cycles of heating and cooling to check the metastable and stable phases in the solidified AKD from its melt. The heating or cooling rate employed was $3{ }^{\circ} \mathrm{C} \min ^{-1}$.

XRD patterns of the solidified AKD samples were recorded at several temperatures and time intervals by a powder X-ray diffractometer (RINT 2200, Rigaku) equipped with a sample temperature controller. Monochromatic $\mathrm{Cu}-\mathrm{K} \alpha(0.15418 \mathrm{~nm})$ radiation was obtained 
by a $\mathrm{Ni}-\mathrm{K}_{\beta}$-filter in combination with an electronic pulse-height discriminator. The sample holder $(25 \mathrm{~mm} \times 17 \mathrm{~mm} \times 2 \mathrm{~mm})$ was made of copper to ensure good heat conductivity. The temperature of the sample was controlled within $\pm 0.5{ }^{\circ} \mathrm{C}$. The $\mathrm{X}$-ray diffraction measurements were performed between $1^{\circ}$ and $40^{\circ}$ of $2 \theta$-angle with a counting time of $1 \mathrm{~s}$ for each step of $0.02^{\circ}$. The $2 \theta$-angle was calibrated with copper.

\section{Results}

\subsection{Heat-treatment of AKD surfaces and time-dependent contact angles}

Heat-treatment of each AKD surface was performed at a specified temperature in an incubator. A series of time-dependent contact angles of water droplets on the AKD surfaces of above were determined at room temperature. The results on the AKD surfaces at several curing temperatures are presented in Figures 2 and 3. The increase in the contact angle value against time together with some photographs of the water droplets clearly shows the progressive formation of the super water-repellent surfaces. The contact angle increases steeply during a certain period of heat-treatment. The final stage of this change gives the contact angles higher than $150^{\circ}$ and the surface shows super water-repellency. A water droplet rolls around without attachment on the super water-repellent surfaces. Athough the initial contact angles on the freshly solidified pure or mixed AKD surfaces are almost same, the time requirements for the surfaces to become super water-repellent at various 
temperatures are quite different. It takes about 6 days for the pure AKD surface to show its super water-repellency at $40{ }^{\circ} \mathrm{C}$, but does 1 hour at $50{ }^{\circ} \mathrm{C}$. The mixed AKD surface becomes super water-repellent in 5 days at room temperature, while it takes only 50 minutes at $45{ }^{\circ} \mathrm{C}$. It is concluded that the water-repellency of the AKD surfaces can be thermally induced quite effectively.

\subsection{Fractal structures of the mixed AKD surfaces and their fractal dimension}

SEM images for the mixed AKD surfaces and the cross sectional views of the super water-repellent surface are shown in Figure 4. SEM observations indicate that the fresh mixed AKD surface (4a) just after solidification from its melt has no special structure and most parts of the surface are smooth. The super water-repellent surface (4b), however, exhibits extreme roughness with fractal flake-like structures. The typical images of the cross section with several magnifications $(4 c-4 f)$ show that the thickness of the rough fractal structures on the super water-repellent mixed AKD surface is about $8 \mu \mathrm{m}$ and that of each flake on the surface is about $100 \mathrm{~nm}$. Similar SEM images for the pure AKD surfaces were obtained, but not shown here since they were already published elsewhere. ${ }^{11,12}$

The box-counting method was used to determine the fractal dimension of the super water-repellent AKD surface. The correponding trace curves were obtained with the help of Adobe Photoshop 7.0.1 from the SEM images with different magnifications of the cross 
section. A box-counting program was then used to count the number of boxes with different sizes to cover the trace. The fractal dimension $D_{\text {cross }}$ of the trace curves of the cross section, the box size $r$ and box number $\mathrm{N}(r)$ are in the following relationship.

$$
N(r) \propto r^{-D_{\text {cross }}}
$$

The dimension $D_{\text {cross }}$ can be calculated from the slope of the plot of $\log \mathrm{N}(r)$ versus $\log r$. The fractal dimension $D$ of the surface was then evaluated as $D=D_{\text {cross }}+1 .^{11,12}$ The real scale of the box was obtained from the box-counting size together with the scale bar and pixels of the SEM images.

The trace curves of the super water-repellent mixed AKD surface with fractal geometry are shown in Figure 5. Figure 6 gives the box-counting results for the trace curves in Fig. 5. The slope of the straight line is -1.26 and then the fractal dimension is $D=1.26+1=2.26$, the value of which is similar to that of the fractal pure AKD surface (2.29). ${ }^{11,12}$ It can be seen from Fig. 6 that the super water-repellent mixed AKD surface also exhibits typical fractal nature. Such fractal structures play an important role in the enhancement of the real surface area of the AKD surface and its water-repellency.

\subsection{Phase transition during the formation of fractal super water-repellent AKD surfaces}

DSC and XRD measurements were utilized to investigate the phase transformation and the degree of crystallization of solid AKD. Figure 7 shows the DSC thermograms at heating / cooling rate of $3{ }^{\circ} \mathrm{C} \min ^{-1}$ for the solvent-crystallized (7a, 7d), freshly solidified (7b, 7e) 
and super water-repellent (7c, 7f) AKD samples. One can see a small exothermic peak at 54 ${ }^{\circ} \mathrm{C}(7 \mathrm{~b})$, which correponds to the transformation from the metastable to the stable phase in the pure $\mathrm{AKD}$, or an endothermic band around $43{ }^{\circ} \mathrm{C}(7 \mathrm{e})$, which correponds to the melting process of the metastable phase before the normal melting point of the stable crystalline phase in the mixed AKD, in the DSC curves of the freshly solidified AKD samples. These kinds of phase transformation of the metastable phase disappear in the solvent crystallized (7a, 7d) or the super water-repellent (7c, 7f) AKD samples. In the second cycles of the scanning, however, the three samples of the pure or mixed AKD undergo the same changes as the freshly solidified AKD does. Hence, there surely exists a metastable phase in the fresh surfaces of the pure or mixed AKD prepared by the rapid solidification method from its melt. The thermal anormalies before melting were observed quite clearly in the triglyceride waxes since all the sample specimen transformed once into metastable $\alpha$-form when crystallized from their melts. ${ }^{13}$ Although the anormalies of the AKD samples appeared in Figs. $7 \mathrm{~b}$ and $7 \mathrm{e}$ are small compared with those of the triglycerides, but their behaviors in DSC are essentially the same.

The wide angle XRD patterns of the solidified AKD samples were recorded at several temperatures and time intervals as shown in Figures $8 \mathrm{a}$ and $8 \mathrm{~b}$. After the initial record of XRD pattern at $28{ }^{\circ} \mathrm{C}$, the AKD sample was heated to the next temperature at the heating rate of $2{ }^{\circ} \mathrm{C} \min ^{-1}$ and was kept at that temperature for 10 minutes before measurement. Two separate records were carried out with the time interval of 2 hours at each temperature. As 
shown in Figures $8 \mathrm{a}$ and $8 \mathrm{~b}$, there are many broad reflection peaks at the $2 \theta$-angle range from $16^{\circ}$ to $27^{\circ}$, especially in the patterns at relatively low temperatures. It implies that the freshly solidified AKD samples are, at least partly, in the metastable phase in poorly ordered state. Phase transformations from the metastable state to the stable one of the pure AKD at three different temperatures are shown clearly in Figures 8c, 8d and 8e. The metastable and stable states of the AKD are typically characterized by the reflections at $2 \theta=23.5^{\circ}$ and 24.3 ${ }^{\circ}$, respectively.

The peaks in the XRD patterns of the stable AKD shown in Figures $8 \mathrm{a}$ and $8 \mathrm{~b}$ have almost same positions but obviously different relative intensities. The changes of the relative intensities of the most important reflections in the XRD patterns are presented in Figure 9. As shown in Figures 8 and 9, with the increase of the temperature, the peaks become sharper and the intensities become stronger in the aged samples than those in the fresh one, which indicates that the degree of crystallization increases after the phase transformation from the metastable state to the stable crystalline one.

\section{Discussion}

4.1 Mechanism of spontaneous formation of super water-repellent AKD surfaces with

\section{fractal structures}


We have made clear the mechanism of spontaneous formation of fractal structures on the surfaces of triglyceride waxes. ${ }^{13}$ In this system, the phase transition from the metastable $\alpha$-form to the stable $\beta$-form crystal is essential for spontaneous formation of fractal surfaces. We have observed the quite similar results of the time-dependent contact angles, DSC and XRD experiments also in the present AKD system to those of the triglycerides as shown below. There exists a solid-(liquid)-solid phase transformation from the metastable to the stable crystalline state, as shown in Figures 7, 8 and 9 obtained from the results of DSC and XRD measurements, when a freshly solidified AKD undergoes heat-treatment at a specified temperature. The phase transformations, typically shown in Figure $8 \mathrm{c}, 8 \mathrm{~d}$ and $8 \mathrm{e}$, take place spontaneously because the Gibbs enegy of the metastable state is always higher than that of the stable one at the same temperature, and the rate of the phase changes depends strongly upon the temperature. Furthermore, the XRD results together with the DSC curves shown in Figures $9 \mathrm{~b}$ and $9 \mathrm{~d}$ indicate clearly that the relative intensities, especially of the short spacing peaks, increase steeply with the temperature at which the pre-melting or the phase transition takes place.

As mentioned in the experimental section, the rapid solidification from the melt leads to the formation of a metastable phase in poorly ordered state in the fresh AKD surface. Under heat-treatment, the AKD molecules in the metastable phase get enough energy of motion to arrange themselves spontaneously into the thermodynamically favorable crystalline form. Higher temperature can effectively accelerate the molecular rearrangement and the structure 
adjustment, and then enhance the phase transformation from the metastable to the stable crystalline state. These thermally induced molecular orientations promote the formation of microcrystals. With the formation of the stable microcrystals on the surface, the fractal structures gradually grow, the surface becomes rough, and finally does super water-repellent. The increase in contact angle can then be observed at a given temperature. Hence, the spontaneous formation of super water-repellent fractal surface of AKD results from the phase transformation from the metastable to the stable cystalline state. Appropriate heat-treatment shows significant effects on the rate of phase transformation and the spontaneous formation of super water-repellent surface with fractal structures.

\subsection{Activation energy of spontaneous formation of super water-repellent AKD surfaces}

We define $t_{150}$ as the time required for a solid surface to become super water-repellent with the water contact angle of $150^{\circ}$ at a given temperature. From the Arrhenius equation

$$
k=A e^{-E_{a} / R T}
$$

we have

$$
\ln \left(1 / t_{150}\right)=-\left(\frac{E_{a}}{R}\right) \frac{1}{T}+C
$$

where $k$ is the rate constant, $A$ the pre-exponential factor, $E_{\mathrm{a}}$ the activation energy, $R$ the gas

constant, $T$ the absolute temperature and $C$ is a constant.

The plots of $\ln \left(1 / t_{150}\right)$ versus $1 / T$ are given in Figure 10, where $t_{150}$ is in $\mathrm{h}$ and $T$ is in $\mathrm{K}$. From the slopes of the straight lines, the activation energies for the pure and mixed AKD are 
obtained to be 415 and $158 \mathrm{~kJ} \mathrm{~mol}^{-1}$, respectively. These values indicate quantitatively the energy requirements of the thermally activated transformation from an initial AKD surface with water contact angle lower than $120^{\circ}$ to a super water-repellent one with the contact angle larger than $150^{\circ}$.

\section{Conclusions}

Inspired by the fractal structures and the super water-repellent behavior of the triglyceride surfaces, ${ }^{13}$ we have investigated the mechanism of spontaneous formation of super water-repellent fractal surfaces of alkylketene dimer (AKD). The fractal structures on the AKD surfaces also form spontaneously during the processes of the phase transformation from the metastable to the stable crystalline phase and the growth of a lot of stable microcrystals. The super water-repellency is thermally induced quite effectively. The time requirement for the water contact angle of $150^{\circ}$ on the AKD surface decreases exponentially with the increase of the curing temperature. The activation energies for the formation of super water-repellent fractal AKD surfaces can be evaluated quantitatively to be 415 and $158 \mathrm{~kJ} / \mathrm{mol}$ for the pure and mixed $\mathrm{AKD}$, respectively from the time-dependent contact angle measurements. 


\section{Acknowledgement}

We are grateful for the financial support from 21st century COE program "Center of

Excellence for Advanced Life Science on the Base of Bioscience and Nanotechnology" of Hokkaido University, Japan, and a Grant-in-Aid for Scientific research (B) (No. 16310077) from the Ministry of Education, Culture, Sports, Science and Technology, Japan. 


\section{References}

[1]M. Callies and D. Quéré, Soft Matter 1 (2005) 55.

[2] W. Barthlott and C. Neinhuis, Planta 202 (1997) 1-8.

[3] W. Chen, A. Y. Fadeev, M. C. Hsieh, D. Öner, J. Youngblood and T. J. McCarthy, Langmuir 15 (1999) 15, 3395-3399.

[4] D. Öner and T. J. McCarthy, Langmuir 16 (2000) 7777-7782.

[5] L. Gao and T. J. McCarthy, Langmuir 22 (2006) 5998-6000.

[6] K. Tsujii, in Surface Activity_Principles, Phenomena, and Applications, ed. T. Tanaka, Academic press, New York, 1998, pp. 52-54.

[7] A. Nakajima, A. Fujishima, K. Hashimoto and T. Watanabe, Adv. Mater. 11 (1999) $1365-1368$.

[8] J.-Y. Shiu, C. -W. Kuo, P. Chen and C. -Y. Mou, Chem. Mater. 16 (2004) 561-564.

[9] C. Sanchez, H. Arribart, M. M. G. Guille, Nature Mater. 4 (2005) 277-288.

[10] G. McHale, M. I. Newton and N. J.Shirtcliffe, European J. Soil Sci. 56 (2005) 445-452.

[11] T. Onda, S. Shibuichi, N. Satoh and K. Tsujii, Langmuir 12 (1996) 2125-2127.

[12] S. Shibuichi, T. Onda, N. Satoh and K. Tsujii, J. Phys. Chem. 100 (1996) 19512-19517.

[13] W. Fang, H. Mayama and K. Tsujii, J. Phys. Chem. B 111 (2007) 564-571.

[14] R. Mohammadi, J. Wassink and A. Amirfazli, Langmuir 20 (2004) 9657-9662.

[15] K. Tadanaga, N. Katata and T. Minami, J. Am. Ceram. Soc. 80 (1997) 1040-1042. 
[16] A. Nakajima, K. Abe, K. Hashimoto and T. Watababe, Thin Solid Films 376 (2000) $140-143$.

[17] K. Takeda, M. Sasaki, N. Kieda, K. Katayama, T. Kako, K. Hashimoto, T. Watanabe and A. Nakajima, J. Mater. Sci. Lett. 20 (2001) 2131-2133.

[18] A. Hozumi and O. Takai, Thin Solid Films 303 (1997) 222-225.

[19] D. O. H. Teare, C. G. Spanos, P. Ridley, E. J. Kinmond, V. Roucoules and J.P.S. Badyal, Chem. Mater. 14 (2002) 4566-4571.

[20] H. Y. Erbil, A. L. Demirel, Y. Avci and O. Mert, Science 299 (2003) 1377-1380.

[21] K Tsujii, T. Yamamoto, T. Onda and S. Shibuichi, Angew. Chem. Int. Ed. 36 (1997) 1011-1-12.

[22] S. Shibuichi, T. Yamamoto, T. Onda and K. Tsujii, J. Colloid Interface Sci. 208 (1998) 287-294.

[23] N. J. Shirtcliffe, G. McHale, M. I. Newton and C. C. Perry, Langmuir 21 (2005) 937-943.

[24] H. Yan, K. Kurogi, H. Mayama and K. Tsujii, Angew. Chem. Int. Ed. 44 (2005) 3453-3456.

[25] N. Zhao, F. Shi, Z. Wang and X. Zhang, Langmuir 21 (2005) 4713-4716.

[26] S. Wang, L. Feng and L. Jiang, Adv. Mater. 18 (2006) 767-770.

[27] M. Y. Yüce, A. L. Demirel and F. Menzel, Langmuir 21 (2005) 5073-5078.

[28] C.-T. Hsieh, J.-M. Chen, R.-R. Kuo, T.-S. Lin and C.-F. Wu, Appl. Surf. Sci. 240 (2005) 318-326.

[29] J. Zhang, W. Huang and Y. Han, Langmuir 22 (2006) 2946-2950. 
[30] S. Li, H. Li, X. Wang, Y. Song, Y. Liu, L. Jiang and D. Zhu, J. Phys. Chem. B 106 (2002) 9274-9276.

[31] X. Feng, L. Feng, M. Jin, J. Zhai, L. Jiang and D. Zhu, J. Am. Chem. Soc. 126 (2004) $62-63$.

[32] L. Feng, Y. Song, J. Zhai, B. Liu, J. Xu, L. Jiang and D. Zhu, Angew. Chem. Int. Ed. 42 (2003) 800-802.

[33] T. Sun, G. Wang, H. Liu, L. Feng, L. Jiang and D. Zhu, J. Am. Chem. Soc. 125 (2003) 14996-14997.

[34] L. Feng, Z. Yang, J. Zhai, Y. Song, B. Liu, Y. Ma, Z. Yang, L. Jiang and D. Zhu, Angew. Chem. Int. Ed. 42 (2003) 4217-4220.

[35] M. Jin, X. Feng, L. Feng, T. Sun, J. Zhai, T. Li and L. Jiang, Adv. Mater. 17 (2005) 1977-1981.

[36] E. Martines, K. Seunarine, H. Morgan, N. Gadegaard, C. D. W. Wilkinson and M. O. Riechle, Nano Lett. 5 (2005) 2097-2103.

[37] P.S. Swan and R. Lipowsky, Langmuir 14 (1998) 6772-6780.

[38] M. Miwa, A. Nakajima, A. Fujishima, K. Hashimoto and T. Watanabe, Langmuir 16 (2000) 5754-5760.

[39] N. A. Patankar, Langmuir 19 (2003) 1249-1253.

[40] J. Bico, C. Marzolin, D. Quéré, Europhys. Lett. 47 (1999) 220-226.

[41] J. Bico, C. Tordeux and D. Quéré, Europhys. Lett. 55 (2001) 214-220. 
[42] J. Bico, U. Thiele and D. Quéré, Colloids Surf. A 41 (2002) 41-46.

[43] D. Quéré, Physica A 313 (2002) 32-46.

[44] A. Marmur, Langmuir 20 (2004) 3517-3519.

[45] C. W. Extrand, Langmuir 22 (2006) 1711-1714.

[46] R. D. Hazlett, J. Colloid Interface Sci. 137 (1990) 527-533.

[47] H. Yan, H. Shiga, E. Ito, T. Nakagaki, S. Takagi, T. Ueda and K. Tsujii, Colloids Surf. A 284-285 (2006) 490-494.

[48] H. Mayama and K. Tsujii, J. Chem. Phys. 125 (2006) 124706. 


\section{Figure caption}

Fig1. Molecular structure of pure alkylketene dimer (AKD).

Fig2. Time-dependent contact angles and some photographs of water droplets on the pure AKD surfaces cured at different temperatures.

Fig3. Time-dependent contact angles and some photographs of water droplets on the mixed AKD surfaces cured at different temperatures. Inset is the changes at the four higher temperatures.

Fig4. SEM images of the freshly solidified (4a) and super water-repellent (4b) surfaces of the mixed AKD, and those of the cross sectional views of the super water-repellent surface $(4 \mathrm{c}-$ $4 f)$.

Fig5. Trace curves of the cross section for the super water-repellent mixed AKD surface obtained from the SEM observations with different magnifications.

Fig6. Plots of $\log N(r)$ versus $\log r$ for the cross sectional trace curves of the super 
water-repellent fractal surface of the mixed AKD. $r$ is the box size and $N(r)$ is the corresponding box number.

Fig.7 DSC thermograms at heating / cooling rate of $3{ }^{\circ} \mathrm{C} \min ^{-1}$ for the pure $(7 \mathrm{a}-7 \mathrm{c})$ and mixed (7d-7f) AKD samples; the solvent recrystallized (7a, 7d), freshly solidified (7b, 7e) and super water-repellent (7c, 7f) AKD. Each sample was scanned for two cycles of heating and cooling. Arrows indicate that $\rightarrow$, heating, $\leftarrow$, cooling, and $\downarrow$, endothermic peak, $\uparrow$, exothermic peak.

Fig.8 XRD patterns of the pure (8a) and mixed (8b) AKD samples at several temperatures and time intervals. The initial record was performed at $28{ }^{\circ} \mathrm{C}$. The sample was then heated to the next temperature at the heating rate of $2{ }^{\circ} \mathrm{C} \min ^{-1}$ and was kept at that temperature for 10 minutes before measurement. Two separate records were carried out with the time interval of 2 hours at each temperature. Figures $8 \mathrm{c}, 8 \mathrm{~d}$ and $8 \mathrm{e}$ show the phase transition from the metastable to the stable state in the solid pure $\mathrm{AKD}$ at $40{ }^{\circ} \mathrm{C}(8 \mathrm{c}), 50{ }^{\circ} \mathrm{C}(8 \mathrm{~d})$ and $59{ }^{\circ} \mathrm{C}(8 \mathrm{e})$.

Fig.9 Relative intensities of several XRD peaks for the pure (9a, 9b) and mixed (9c, 9d) AKD samples as a function of time together with temperature program $(9 a, 9 c)$ and those as a function of temperature along with the DSC curves $(9 b, 9 d)$. 
Fig10. Plots of $\ln \left(1 / t_{150}\right)$ versus $1 / T$ for spontaneous formation of the super water-repellent fractal AKD surfaces. $T$ is the curing temperature in $\mathrm{K} . t_{150}$ in $\mathrm{h}$ is the time requirement for the surfaces with the water contact angle of $150^{\circ}$. 


\section{$\mathrm{R}-\mathrm{CH}=\mathrm{C}-\mathrm{CH}-\mathrm{R}$$$
\mathrm{R}=n-\mathrm{C} 16
$$

Fig. 1 Fang et al. 


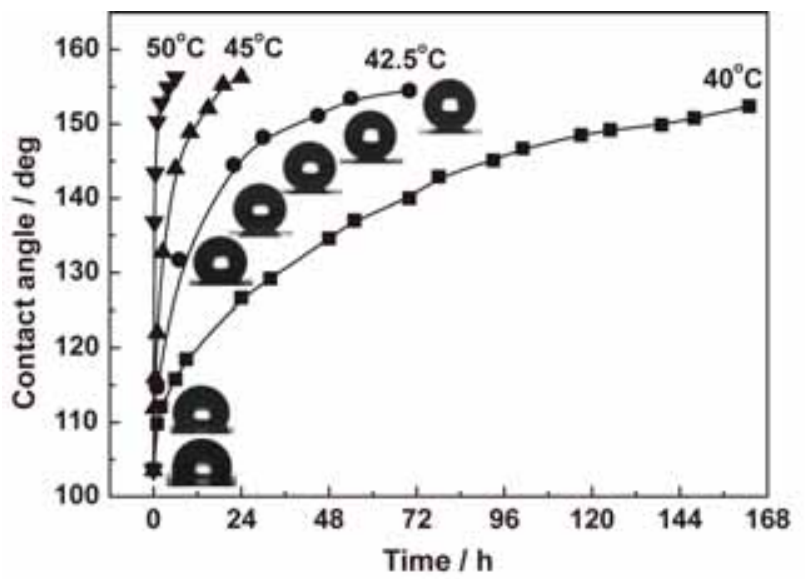

Fig. 2 Fang et al. 


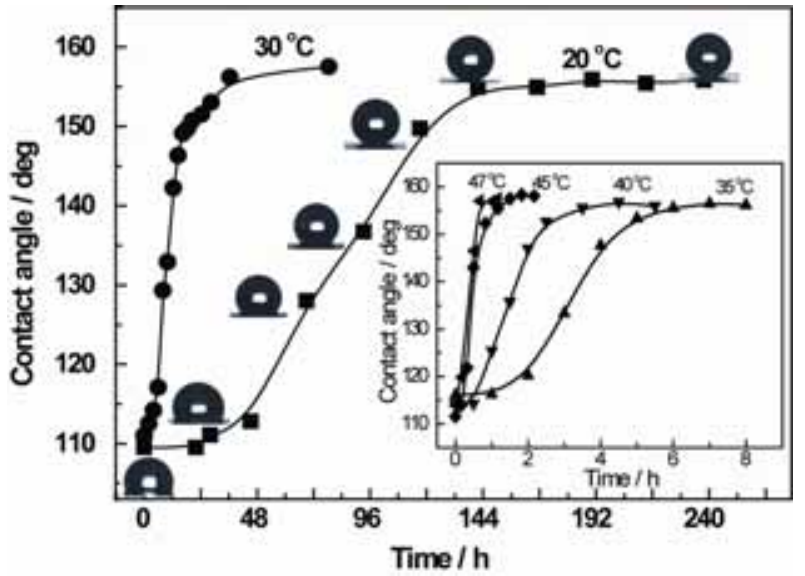

Fig. 3 Fang et al. 


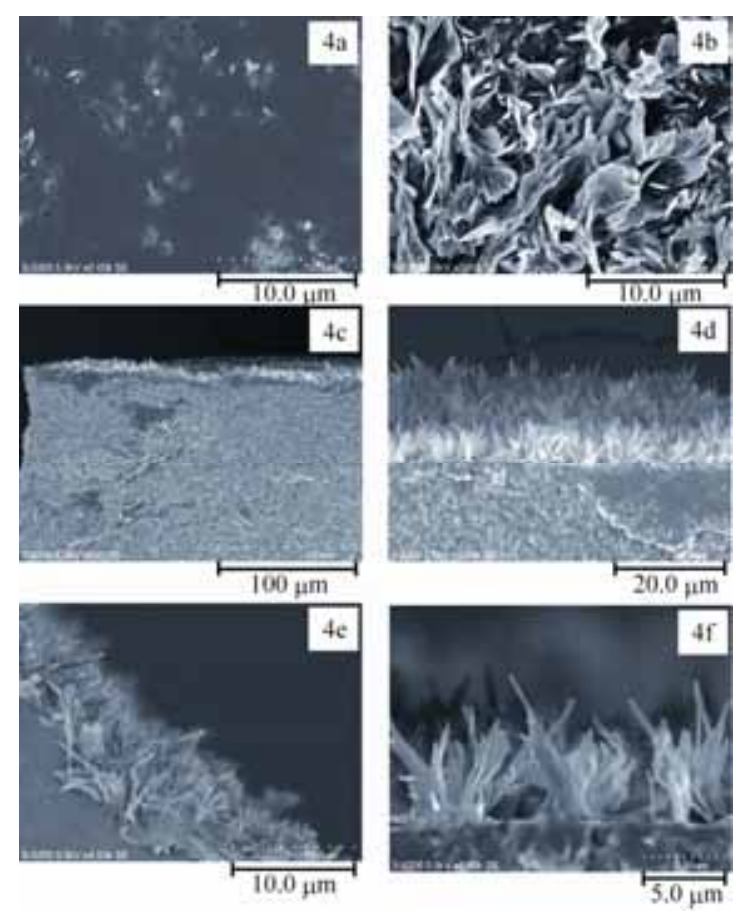

Fig. 4 Fang et al. 


\section{$\times 150$}

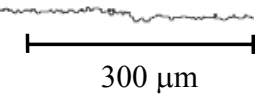

X500
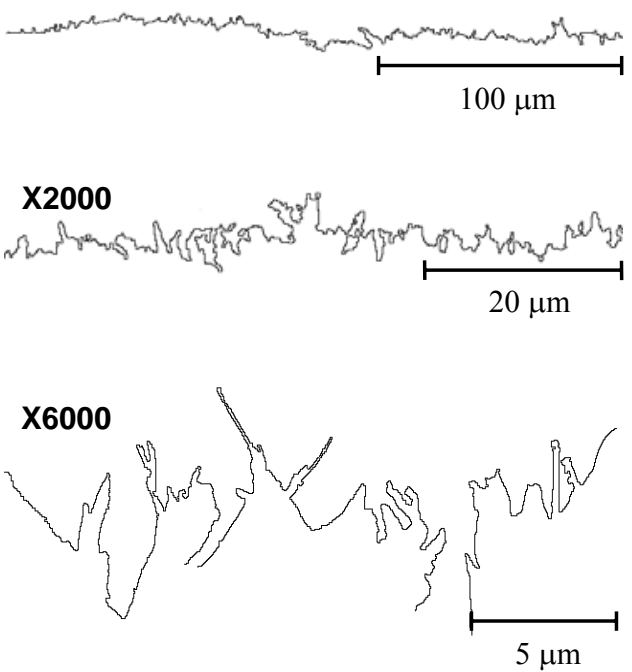

Fig. 5 Fang et al. 


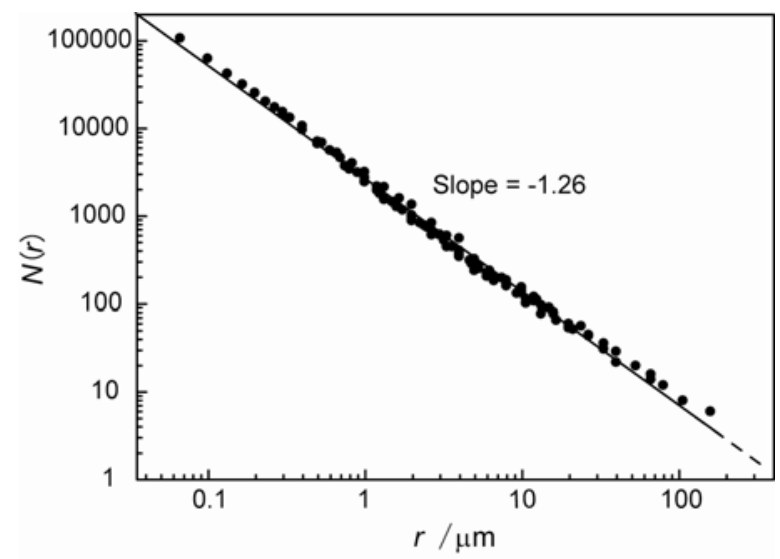

Fig. 6 Fang et al. 

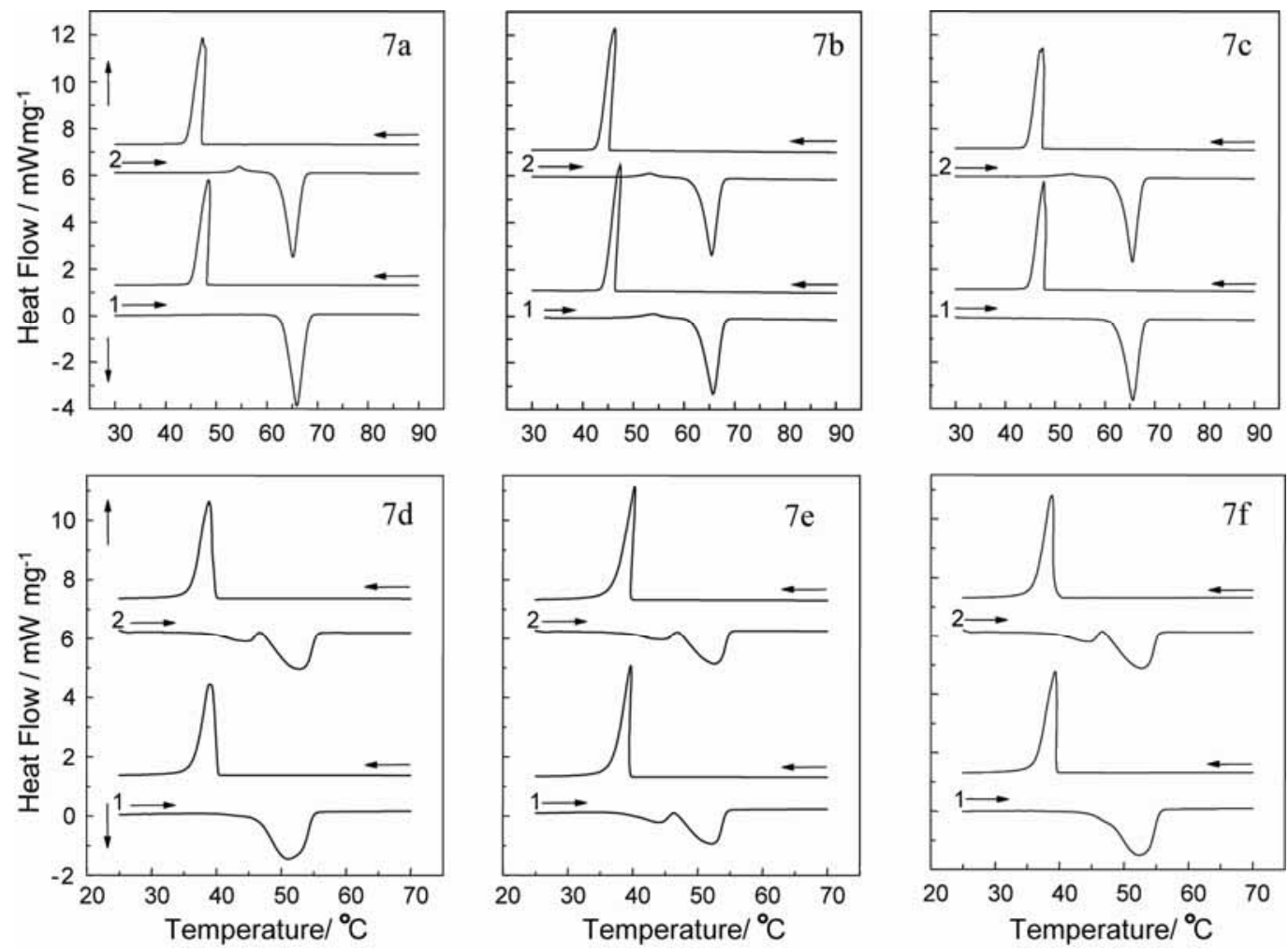

Fig. 7 Fang et al. 

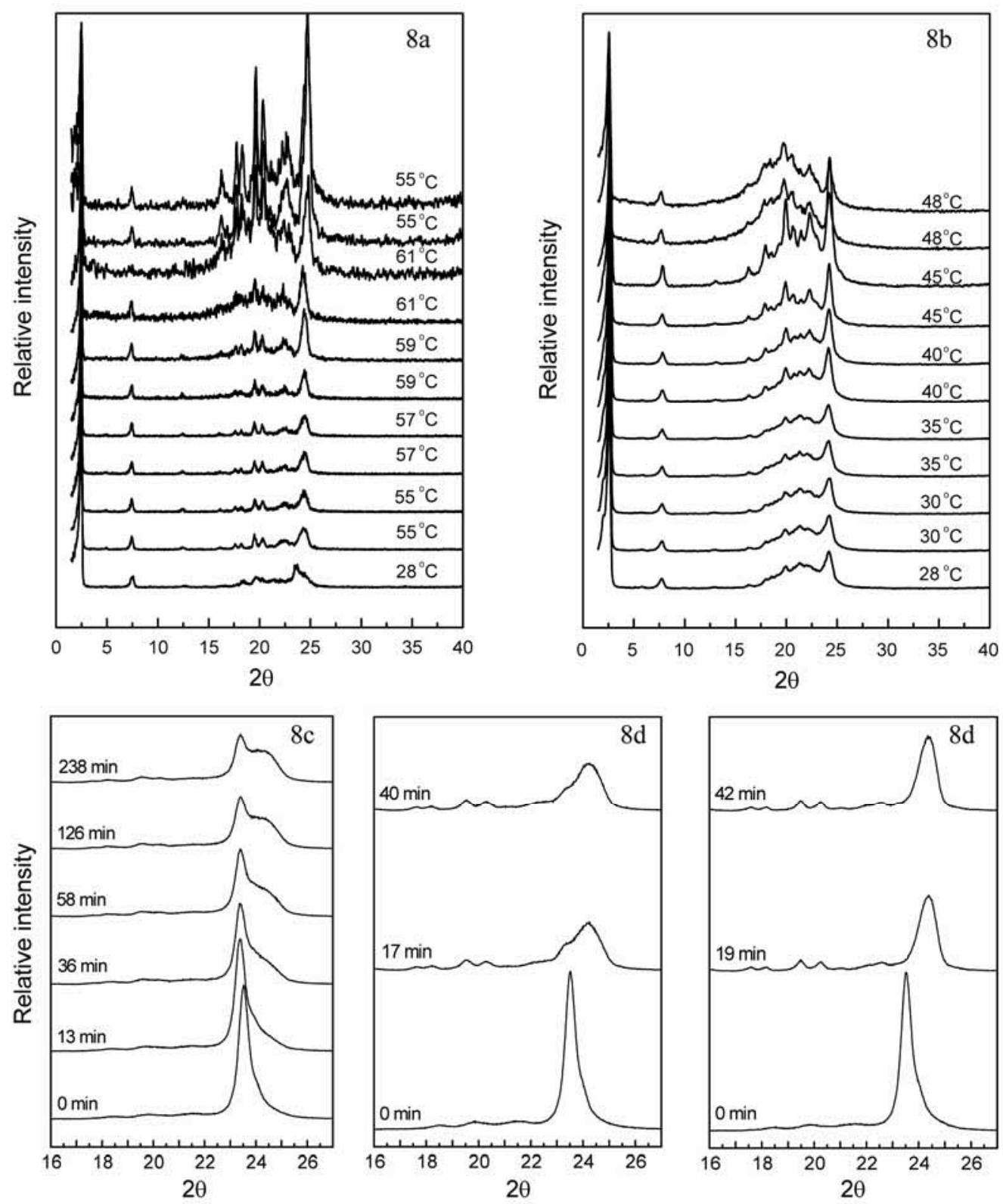

Fig. 8 Fang et al. 

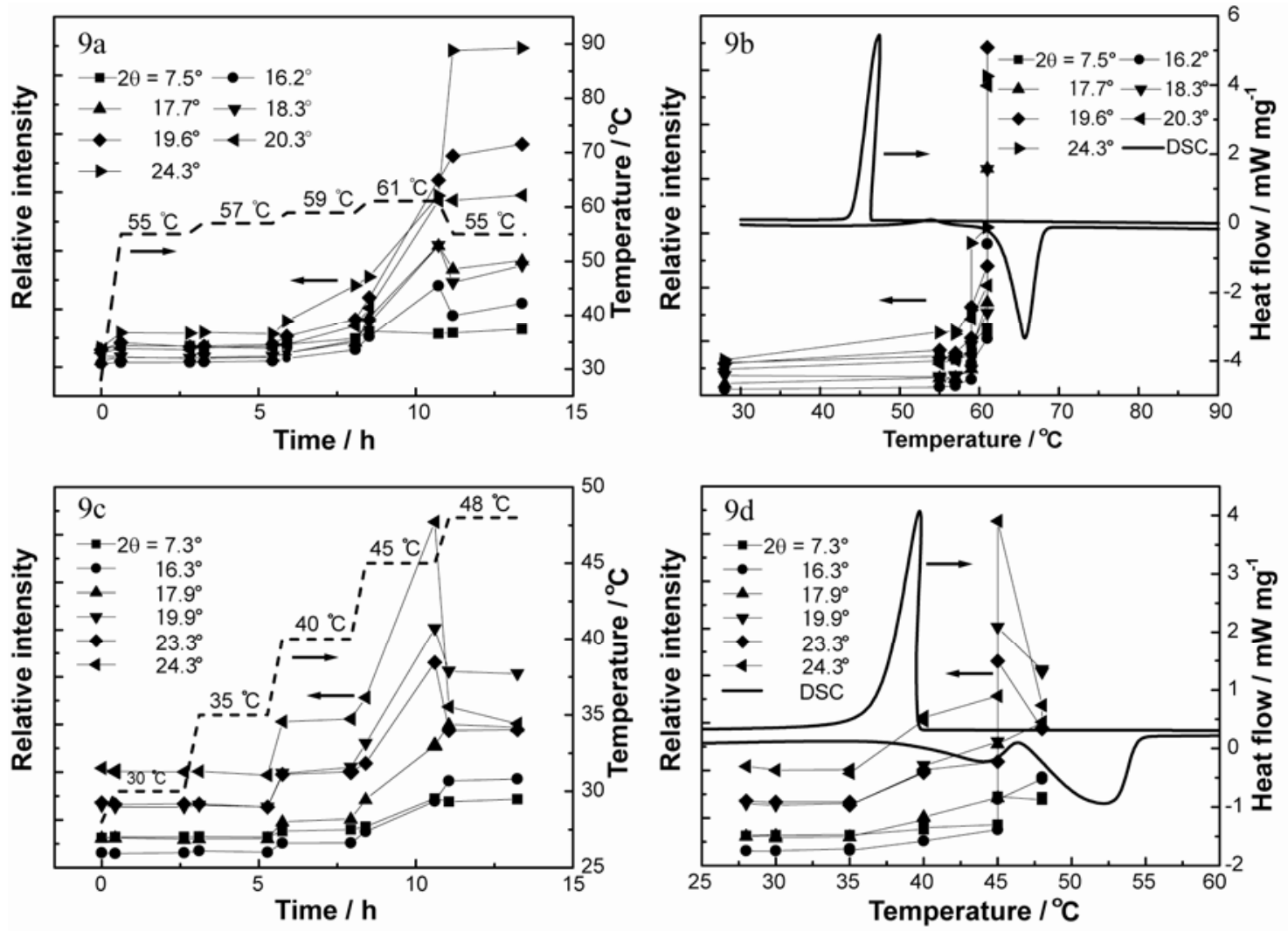

Fig. 9 Fang et al. 


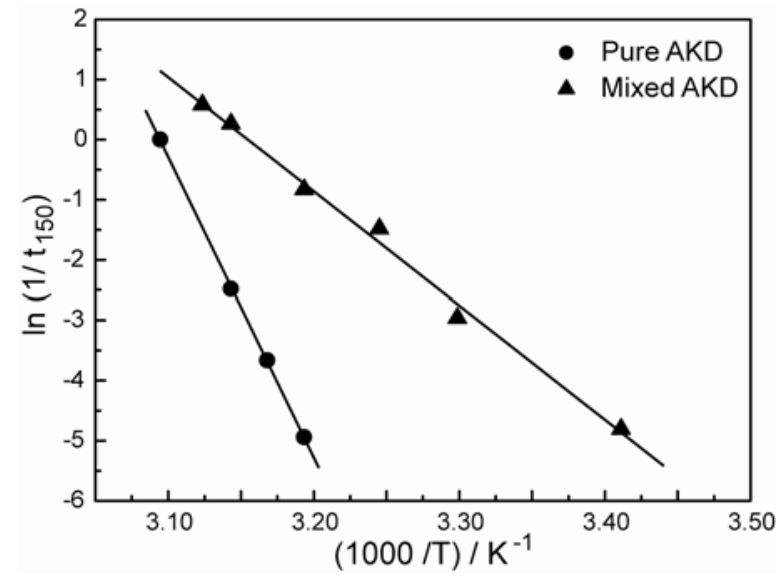

Fig. 10 Fang et al. 